\title{
Harmonic synchronization model of the mating dengue vector mosquitoes
}

\author{
YANG Nan, LONG ZhangCai* \& WANG Fei \\ College of Physics, Huazhong University of Science and Technology, Wuhan 430074, China
}

Received February 16, 2012; accepted May 2, 2012

\begin{abstract}
When two mosquitoes meet to mate, each modulates its flight tones such that the female's 3rd and the male's 2nd harmonic frequencies are equivalent. We show that this phenomenon is an example of synchronization, which is common in nature. The mosquito's flight tone acts as an external signal, stimulating its partner to adjust the wing beat rhythm to achieve the synchronization state. A simplified model, which is based on the frequency ratio difference feedback mechanism, is proposed to describe the harmonic convergence of mosquitoes. Furthermore, we proposed a method to characterize the energy dissipation in the frequency alteration, and the results demonstrate that $3 / 2$ frequency locking is an optimal selection to mosquitoes. When compared with other possible ratios, the mosquitoes expend minimum energy if they lock the synchronizing state at a ratio of $3 / 2$.
\end{abstract}

frequency locking, feedback, circle map

Citation: $\quad$ Yang N, Long Z C, Wang F. Harmonic synchronization model of the mating dengue vector mosquitoes. Chin Sci Bull, 2012, 57: 4043-4048, doi: $10.1007 / \mathrm{s} 11434-012-5445-\mathrm{Z}$

As the disease carrier of malaria, yellow fever and dengue, mosquitoes, and their mating mechanics in particular, have been popular topics of research. Since Johnston discovered mosquito's hearing organ and speculates that it is related to the mating behaviors [1], much insight has been gained into the auditory mechanics and their roles in sexual recognition [2-4]. Recently, a remarkable behavioral experiment on the mating of dengue vector mosquitoes was conducted by $\mathrm{Ca}-$ tor et al. [5]. As two heterosexual mosquitoes enter one another's hearing range, both of them adjust their wing beat frequencies to a harmonic convergence. What is counterintuitive, however, is that the convergence occurs neither at the fundamental frequency of the female $(430.6 \mathrm{~Hz})$ nor that of the male $(636.7 \mathrm{~Hz})$, but rather at a tone $(1350 \mathrm{~Hz})$ near the female's 3rd harmonic and the male's 2nd harmonic. Nevertheless, the mechanical reason for two mosquitoes fixing on the special frequency ratio of $3 / 2$ is still unknown. In this work, we consider a simplified model to describe the phenomenon of $3 / 2$ harmonic convergence. Based on this model, we show that the frequency ratio of $3 / 2$ is an optimal

*Corresponding author (email: longzc01@mails.tsinghua.edu.cn) selection for mosquitoes.

The harmonic convergence of mosquitoes observed in the experiment can be understood and studied within the framework of phase synchronization (frequency locking). The synchronization phenomenon, first discovered in a simple double pendulum system [6], has been observed in a variety of complex living organisms [7-18], such as plant leaves, swimming microorganisms and bursting neurons. It is generally acknowledged that the interactions of living individuals lead to the emergence of synchronization. However, in previous studies the living individuals were considered as passive coupling oscillations, pursuant to given rules for action coherence. The active tuning mechanism, which exists widely in biological systems, has rarely been mentioned in previous mathematic descriptions of synchronization behaviors. Our model, based on the active "frequency difference" feedback mechanism [19], will qualitatively describe the harmonic synchronization of two mating mosquitoes. In this work, we utilize the circle map theory to simulate the evolution of the wing beat frequencies in the mating mosquitoes. Circle map describes the motion of two coupling oscillations only with two parts: the ratio of 
natural frequencies and the coupling item. We introduced the feedback theory into the coupling item, which lead to the frequency locking of mating mosquitoes. Therefore we simplify the mosquitoes' frequency adjustment as controlling circle map to a target ratio of frequency locking. The results show, for the first time, the role of energy dissipation in the frequency selection of the mating mosquitoes.

Before introducing our model, the "harmonic convergence" experiment of two mating mosquitoes [5] will be presented in detail. The objects of study, 21 pairs of Aedesaegypti (a type of mosquito), were isolated and tethered to the ends of pins. Cator recorded their average natural wing beat frequencies to be 636.7 and $430.6 \mathrm{~Hz}$ for the male and female mosquitoes, respectively. Mosquitoes will not change their flight tone without acoustic stimulation. Next, the mosquitoes were moved into audible range of their heterosexual partners, at which point the acoustic recording reveals that both males and females adjusted their flight tones so that the females' 3rd harmonic and the males' 2nd harmonic converged at an average frequency of $1354.5 \mathrm{~Hz}$. To verify that the mosquitoes' auditory organ is able to respond to these high-frequency tones, Cator performed another experiment: instead of the female's flight tone, a harmonic combination of electronically generated pure tones $(800,1200 \mathrm{~Hz}$ but lacking the fundamental frequency 400 $\mathrm{Hz}$ ) was played back to male mosquitoes. The results demonstrated that the male mosquito will continue to synchronize its 2nd harmonic frequency to the electronically generated simulated female's 3rd harmonic frequency (1200 $\mathrm{Hz}$ ). The aforementioned experiment revealed that the mosquitoes adjust their flight tone to fix on a specific frequency ratio, rather than a specific frequency.

\section{Model}

To simplify the analysis, in the following discussions, we will focus only on the experiment in which the male adjusts its 2nd harmonic to synchronize the 3rd harmonic of electronic tone. The target frequency ratio is determined by the natural properties of mosquitoes at the very beginning of mating [4,19], and the inner causes are still unknown. However, the latest physiological experiment [19] shows that the mating mosquitoes are especially sensitive to the low frequencies, and they keep tracking and eliminating the low harmonic frequency difference between the frequency pairs. We call it the "active frequency difference feedback mechanism". In Figure 1, the male uses its sense of hearing, which has been demonstrated to have nanometer-range sensitivity [20], to identify the distinction between the 2nd harmonic of its wing beat frequency and the 3rd harmonic of the electronic tone stack; then under the internal adjustments, continuous feedback is applied to the flight frequency so that it would reach the target frequency $[4,19]$. Here, we characterize the period motion of the male mosquito by

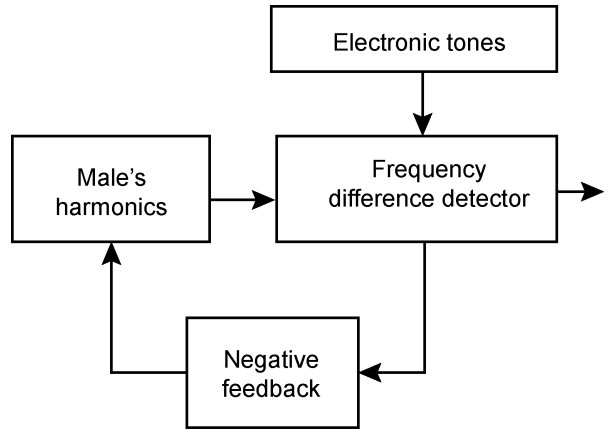

Figure 1 The male mosquito synchronizes its harmonic frequency to the electronic tone.

the phase $\theta(t)$, and thus the instantaneous frequency $f(t)$ can be represented by the change rate of $\theta(t)$ (note: $\theta(t)$ grows monotonously along the time vector and gains 1 after each wing beat period). In our model, we use an equal criterion, the difference $\Delta \rho(t)$ between the instantaneous frequency ratio $\rho(t)$ and the target frequency ratio $\rho_{\text {target }}$, to detect the synchronization state. Based on this "active frequency ratio difference feedback mechanism", a linear integral feedback item is designed and combined into the mathematical model of the evolving of wing beat phase $\theta(t)$. If the frequency ratio difference $\Delta \rho(t), \Delta \rho(t)=\rho(t)-\rho_{\text {target }}$, is not equal to zero, continuous adjustment will feed back to the wing beat phase $\theta(t)$ to ensure that the male will reach the synchronization state.

The evolution of phase $\theta(t)$ is modeled as a feedback phase oscillation, which evolves with its natural frequency $f_{1}$ and modulates itself according to the feedback component $Q(\theta, t)$ :

$$
\frac{\mathrm{d} \theta}{\mathrm{d} t}=f_{1}+Q(\theta, t)
$$

$Q(\theta, t)$ is defined as the function of the instantaneous frequency ratio $\rho(t)$ between the male mosquito and the electronic tone:

$$
\rho(t)=\frac{1}{f_{2}} \frac{\mathrm{d} \theta}{\mathrm{d} t} .
$$

A stroboscopic mapping is used on eqs. (1) and (2) with the time interval $T=1 / f_{2}$, which gives the evolving of $\theta(t)$ in the form of circle map [21-23]:

$$
\theta(n+1)=\theta(n)+\Omega+F(n),
$$

where $\Omega=f_{1} / f_{2}$, denotes the natural frequency ratio of male and electrical tone. Here, we use $\rho\left(t_{n}\right)$ to denote the discretized instantaneous frequency ratio $\rho(t), t_{n}=t_{0}+n T . \rho\left(t_{n}\right)$ is approximately represented by the average frequency ratio among $\left[t_{0}+n T-m T, t_{0}+n T\right]$ in eq. (1):

$$
\rho\left(t_{n}\right)=\frac{1}{f_{2}} \frac{\theta\left(t_{0}+n T\right)-\theta\left(t_{0}+n T-m T\right)}{m T} .
$$


When $T \rightarrow 0, \rho\left(t_{n}\right) \rightarrow \rho(n T)$. The instantaneous frequency ratio $\rho(n)$ in circle map is

$$
\rho(n)=\frac{\theta(n)-\theta(n-m)}{m} .
$$

The feedback component $F(n)$ is designed as the sum of frequency ratio differences $\Delta \rho(i)$ with different proportional constants $k_{i}, i=1,2, \ldots n$ :

$$
F(n)=-\sum_{i=m}^{n} k_{i}\left(\rho(n)-\rho_{\text {target }}\right) .
$$

We set $m=1$ to minimize the deviation in frequency ratio $\rho(n)$. Let $k_{1}=k_{2}=\ldots=k_{n}=k$, which gives a simplified form of feedback item $F(n)$ :

$$
F(n)=-k\left(\theta(n)-n \rho_{\text {target }}\right)
$$

Combining eqs. (6) and (7), we have

$$
\theta(n+1)=(1-k) \theta(n)+\Omega+n k \rho_{\text {target }} .
$$

Here, $\theta(n)$ depends on three parameters: the gain $k$, the natural frequency ratio $\Omega$, and the target ratio $\rho_{\text {target }} k$ and $\Omega$ are prescribed values, while the selection of $k$ determines the convergence rate and the stability of system (8) [24]. The closed-loop system matrix of system (6) is represented by $I=1-k$ and the corresponding characteristic polynomial $\Delta s=$ $s-1+k$. System (6) is considered to be asymptotic stable if and only if $|s|<1$. Therefore we have the asymptotic stability region of $k: k \in(0,2)$.

Our purpose is to describe the harmonic convergence of male mosquito and electrical tone; here the male's instantaneous frequency can be represented as $f(n)=f_{2} \rho(n)$. A numerical simulation of our theory is shown in Figure 2(a) and (b); the male's 2nd harmonic frequency gradually approaches the simulated 3rd harmonic frequency of a female, which coincides with the results of the corresponding experiment data shown in Figure 2(c). We set the parameter $\rho_{\text {target }}=3 / 2, k=0.5 \times 10^{-4}$. Because $T=1 / f_{2}$ is utilized as the time interval of stroboscopic mapping, $f_{2}=400 \mathrm{~Hz}$, so every 400 iterations equals to one second.

\section{Energy dissipation}

Why, then, do the male mosquitoes not lock at other frequency ratios, such as the ratio $2 / 1$, representing the case in which the male must synchronize its fundamental frequency to the simulated female's 2nd harmonic. Here we will demonstrate that the frequency locking ratio $\rho_{\text {target }}=3 / 2$ is optimal for the mating mosquitoes. Cator also performed a physiological experiment regarding the mosquitoes' auditory organ, the result of which demonstrated that probe tone $2000 \mathrm{~Hz}$ is the peak value to be responded. This frequency, $2000 \mathrm{~Hz}$, falls between the 4th and 5th harmonic of females,
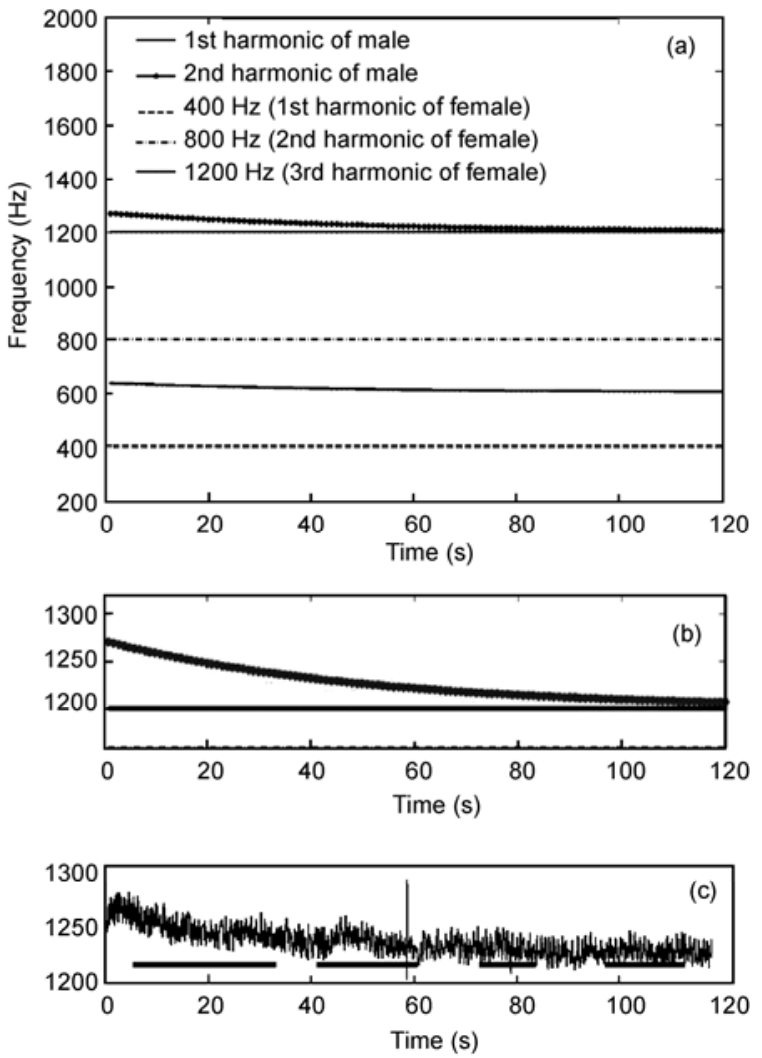

Figure 2 (a) The evolution of the male's wing beat frequency as predicted by our theory; (b) an expand view of (a); (c) the corresponding observed data in Cator's experimental [5].

so we let 4 be the maximum numerator of the target frequency ratio. Now considering all possible target frequency locking ratios of male and electrical tone: 4/1, 3/1, 2/1, 3/2 and $4 / 3$ (note: the ratio $1 / 1$ is removed because the fundamental frequency of the female is absent in the experiment). Then for each ratio, we calculated the transition time from the nature state to the synchronization state with different values of the control gain $k$. Here, the male and electrical tone are considered to be synchronized when $\Delta \rho(n) \leqslant 10^{-2}$.

In Figure 3, the male can realize the fastest convergence when selecting $3 / 2$ as the target frequency ratio. The selection of gain $k$ can influence the convergence rate when fixing to a specific ratio, but for different target ratios, it will not alter the ranking of synchronization transition time. From the nature frequency to the target frequency, the male mosquito adjusts its wing beat frequency to reach the target ratio of frequency locking. For both sexes of mosquitoes, we suppose that the natural frequency corresponds to a stable state in which the mosquitoes exhaust the minimum energy, and any frequency alteration from the stable state will evoke extra energy dissipation [25-27]. We scale this energy dissipation by the feedback item $F(n)$ :

$$
E(n)=\frac{1}{2} \eta(F(n))^{T} F(n),
$$

where $\eta$ is a parameter to characterize the relation between 


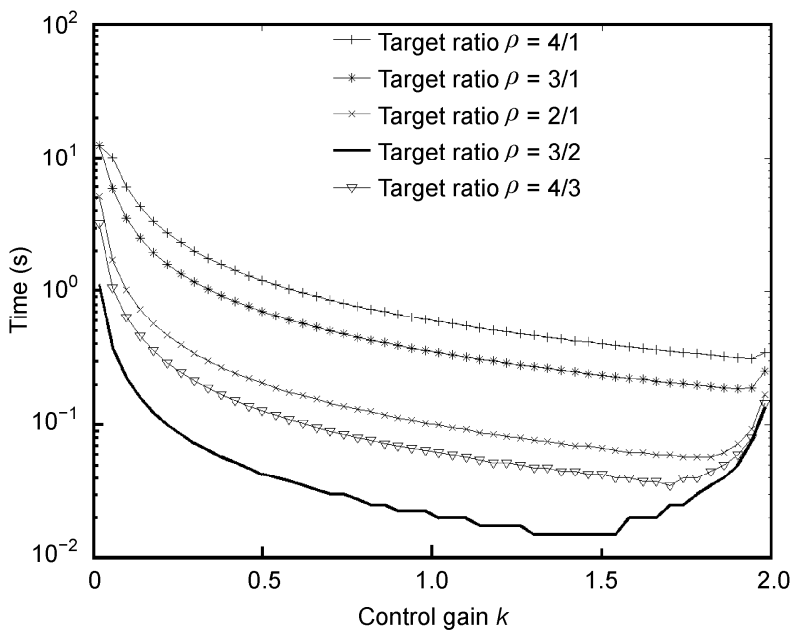

Figure 3 The transition time that from natural frequency to the synchronization state when different target frequency ratio $\rho_{\text {target }}$ and control gain $k$ is tested.

feedback and the real energy dissipation $E(n)$ is the function of $\theta(n)$, which can be solved from eq. (8):

$$
\begin{aligned}
\theta(n)= & (1-k)^{n-1} \theta(1)+\Omega \sum_{i=2}^{n}(1-k)^{i-2} \\
& +k \rho_{\text {target }} \sum_{i=2}^{n}(n+1-i)(1-k)^{i-2}, \quad n \geqslant 2 .
\end{aligned}
$$

Eq. (10) can be transformed into the form

$$
\begin{aligned}
\theta(n)= & (1-k)^{n-1} \theta(1)-k \rho_{\text {target }} \sum_{i=2}^{n}(i-2)(1-k)^{i-2} \\
& +\Omega \sum_{i=2}^{n}(1-k)^{i-2}+(n-1) k \rho_{\text {target }} \sum_{i=2}^{n}(1-k)^{i-2}, n \geqslant 2 .
\end{aligned}
$$

Here, when $n \rightarrow \propto$ and $k \in(0,2)$, we have $\sum_{i=2}^{n}(i-2)(1-k)^{i-2}$

$$
\begin{gathered}
=(1-k) / k^{2} \sum_{i=2}^{n}(1-k)^{i-2}=1 / k . \text { Let } \theta(1)=0, \text { so } \\
\theta(n)=n \rho_{\text {target }}+\left(\Omega-\rho_{\text {target }}\right) / k .
\end{gathered}
$$

Combining eqs. (7) and (12), we have

$$
F(n)=\rho_{\text {target }}-\Omega .
$$

And the energy dissipation when $n \rightarrow \propto$

$$
E(n)=\frac{1}{2} \eta\left(\rho_{\text {target }}-\Omega\right)^{2} .
$$

Above all when $n \rightarrow \infty, k \in(0,2)$, the energy dissipation $E$ is independent of the parameter $k$. It can be easily deduced that when $\rho_{\text {target }}-\Omega=0, E=0$, which coincides with our suppose that mosquitoes exhaust no extra energy when they are in natural state. If the male mosquito dissipates per joule energy in $N$ times wing beat, we have the form of $\eta$ :

$$
\eta=\frac{N}{\left(\rho_{\text {target }}-\Omega\right)^{2}} .
$$

The energy dissipations for different target ratios are explicitly shown in Figure 4; among all the possible ratios, 3/2 frequency locking is optimal. Based on the synchronization behavior of mosquito, we divide the energy dissipation $E$ into two parts: the energy dissipation in transition state $E_{1}$ and the energy dissipation in the frequency locking state $E_{2}$. $E_{1}$ and $E_{2}$ are divided by the transition iterations number $P$, which is shown in Figure 3. $E_{1}$ is the sum of $E(n)$ in the transition process:

$$
E_{1}=\sum_{i=1}^{p} E(i)
$$

And $E_{2}$ is defined as the energy dissipation during $1 \mathrm{~s}$, which equals to $400\left(f_{2}=400 \mathrm{~Hz}\right)$ iterations in the circle map:

$$
E_{2}=\sum_{i=q}^{q+400} E(i), q \succ p .
$$

Because system (8) has converged to the stable state when $n>p$, we have the approximation: $E(n)=E(\propto), n>p$. Thus $E_{2}$ can be simplified as

$$
E_{2}=200 \eta\left(\rho_{\text {target }}-\Omega\right)^{2} .
$$

The value of $\eta$ is difficult to be estimated because the absence of corresponding physiological experiment. However, $\eta$ is a constant value, for each target ratio $\rho_{\text {target }}$, we can calculate the relative energy dissipations, $E_{1} / \eta, E_{2} / \eta$ with various values of $k$. The result is exhibited in Figure $5 ; E_{1}$ and $E_{2}$ were both minimized at the ratio of $3 / 2$ (the bottom line). Beside the case of $3 / 2$, the ratio $4 / 3$ frequency locking (the line marked with triangle) expends the minimum energy, while ratio $4 / 1$ is the one which cost the most. As we

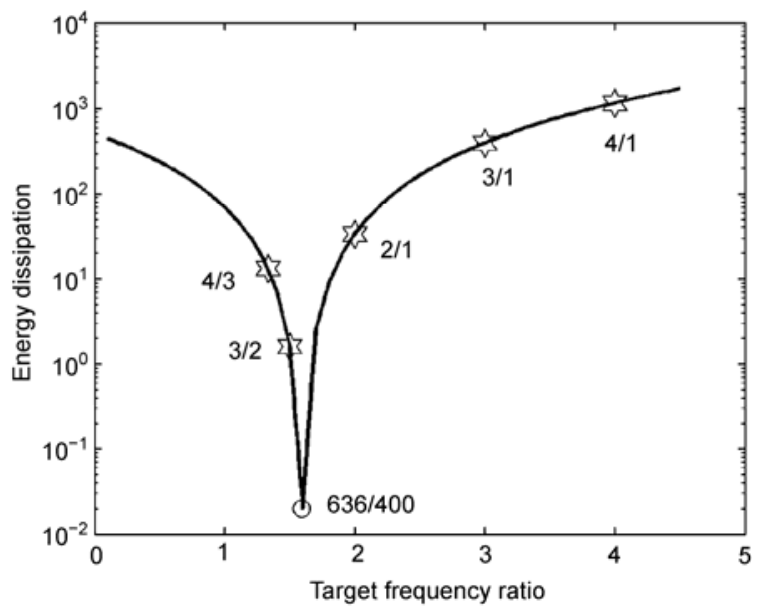

Figure 4 The relative energy spectrum for different target ratios in the stable state. 


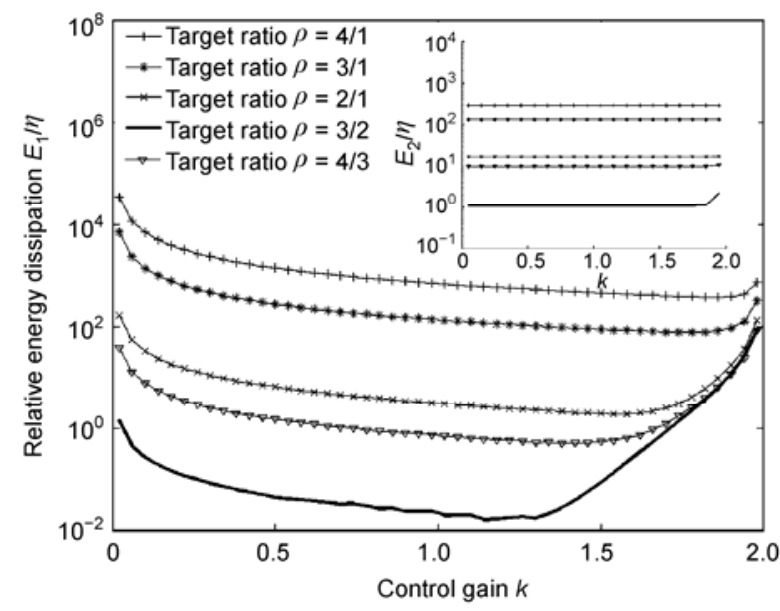

Figure 5 The relative energy dissipation $E_{1} / \eta$ and $E_{2} / \eta$ for different selections of $\rho_{\text {target }}$ and $k$. Note: the first three iterations are removed to reduce the deviation brought by the approximate assumption.

predict in eq. (14), for different values of $k$ in $E_{2}$, the results are almost identical. Thus it can be concluded that the mosquitoes choose a frequency ratio that corresponds to the minimum control energy and fastest convergence, which coincides with the economic principle of nature.

\section{Conclusions and discussion}

We have thus established an active frequency ratio difference feedback based model, which is completely consistent with the experimental evidences. Our theory basis that the negative feedback of frequency difference discrimination leads to the frequency matching of mating mosquitoes, has long been conjectured but proved until very recently [19]. The researches regarding Anopheles gambiae demonstrated that Johnston's organ can detect a low frequency that matches the frequency difference of two combined flight tones, even when the flight tone is beyond the mosquitoes' hearing ranges. This difference-identifying behavior provides continuous feedback that allows the mosquitoes to adjust their flying frequencies to minimize the frequency difference. And A. aegypti, the object of our research, may have the similar mechanisms of low-frequency generation and frequency-difference detection. Even if mosquitoes could accomplish the harmonic matching through acoustic interactions, the hypothesis that mosquitoes can hear the high harmonics remains unproven.

Although Cator emphasized that the fundamental frequency is not necessary for the mating behavior of mosquitoes, the fundamental frequency locking theory we propose in this paper still works because the fundamental frequency is only used as a variable in the harmonic investigation. It should be noted that the 3:2 frequency locking is not a general principle for different species of mosquitoes. For instance, two mating Toxorhynchites Brevipalpis fixed their wing beat frequency on 1:1 [4]. Moreover, the literature shows that the mixed pair of mosquitoes will not stabilize their fundamental frequencies at a fixed ratio, but this experiment only test two different species [19]. For mating pairs, the most advantageous frequency ratio for locking is chosen. The frequency differences between two stacks of harmonics, however, are numerous. How might mosquitoes select the correct frequency difference to eliminate? One persuasive explanation is that the active tuning of mosquitoes auditory organ plays a crucial role in frequency identification. The activity of the Johnston's organ has been studied in [28,29], but how it participate in frequency selection mechanism is still puzzling. As observed in this experiment, a male mosquito can synchronize its flight tone to an electronically generated pure tone that is very different from the component of a female's flight tone. How might the accurate harmonic synchronization behavior be organized by the mosquito? We conjecture that a spontaneous feedbackadjustment mechanism exists that is independent of consciousness in the mating mosquito. From the auditory neurons to muscle neurons, the acoustic information is conducted as electronically generated signals and is finally used to control wing-beating activity.

To summarize, we interpreted the harmonic convergence of mating mosquitoes as the frequency locking of active feedback systems. The harmonic convergence process of mosquitoes has not been considered as a physical model before. Based on the negative frequency ratio difference feedback mechanism, which has been demonstrated in a physiological experiment, we utilized a simplified model to qualitatively describe the harmonic convergence phenomenon. This model successfully described the convergence time and the synchronization orbit of the mating mosquitoes. Furthermore, we demonstrated that the convergence frequency ratio of $3 / 2$ is not an arbitrary value; rather, it corresponds to minimum energy dissipation during the modulation of wing-beat frequency. The frequency ratio selection mechanism, including the active nonlinear amplification in hearing organ and the cooperation between auditory mechanism and wing beat control, would be interesting topics for further experimental studies.

We are grateful to Professor YANG XiaoSong for his illuminating lectures. This work was supported by the National Natural Science Foundation of China (60874099, 90820001).

1 Johnston C, Quart J. Auditory apparatus of the culex mosquito. Microsc Sci, 1855, 3: 97-102

2 Charlwood D M, Jones D R. Mating behaviour in the mosquito, Anopheles gambiae s. l. I. Close range behaviour. Ecol Entomol, 1979, 4: 111-120

3 Belton P J. Attraction of male mosquitoes to sound. Am Mosq Contr Assoc, 1994, 10: 297-301

4 Gibson G, Russell I. Flying in tune: Sexual recognition in mosquitoes. Curr Biol, 2006, 16: 1311-1316

5 Cator L J, Arthur B J, Harrington L C, et al. Harmonic convergence in the love songs of the dengue vector mosquito. Science, 2009, 323: 
1077-1079

6 Huygens C. Horologium Oscillatorium. Parisiis: Apud F. Muguet, 1673

7 Fukuda H, Nakamichi N, Hisatsune M, et al. Synchronization of plant circadian oscillators with a phase delay effect of the vein network. Phys Rev Lett, 2007, 99: 098102

8 Taylor G I. Analysis of the swimming of microscopic organisms. Proc R Soc A, 1951, 209: 447-461

9 Goldstein R E, Polin M, Tuval I. Noise and synchronization in pairs of beating eukaryotic flagella. Phys Rev Lett, 2009, 103: 168103

10 Elfring G, Lauga E. Hydrodynamic phase locking of swimming microorganisms. Phys Rev Lett, 2009, 103: 088101

11 Franovic I, Miljkovic V. Phase plane approach to cooperative rhythms in neuron motifs with delayed inhibitory synapses. Europhys Lett, 2010, 92: 68007

12 Shilnikov A, Gordon R, Belykh I. Polyrhythmic synchronization in bursting network motifs. Chaos, 2008, 18: 037120

13 Vasseur D A, Fox J W. Phase-locking and environmental fluctuations generate synchrony in a predator-prey community. Nature, 2009, 460: $1007-1010$

14 Strogatz S. Sync: The Emerging Science of Spontaneous Order. New York: Hyperion, 2003

$15 \mathrm{Lv}$ L, Li Y, Guo Z A. Parameter identification and synchronization of spatiotemporal chaos in an uncertain Gray-Scott system. Sci China Ser G-Phys Mech Astron, 2008, 51: 1638-1646

16 Yan S L. All-optical and combinational optoelectronic logic gates using chaotic synchronization of coupling-feedback semiconductor lasers and amplitude modulation. Chin Sci Bull, 2011, 56: 1264-1271

17 Ma J, Wu Y, Ying H P, et al. Channel noise-induced phase transition of spiral wave in networks of Hodgkin-Huxley neurons. Chin Sci
Bull, 2011, 56: 151-157

18 Li M S, Zhang H H, Zhao Y, et al. Synchronization of coupled neurons controlled by a pacemaker. Chin Phys Lett, 2011, 28: 010504

19 Pennetier C, Warren B, Dabiré K R, et al. Singing on the wing as a mechanism for species recognition in the malarial mosquito Anopheles gambiae. Curr Biol, 2010, 20: 131-136

20 Gopfert M C, Robert D. Nanometre-range acoustic sensitivity in male and female mosquitoes. Proc Biol Sci, 2000, 267: 453-457

21 Arnold V I. Small denominators. I. Mappings of the circumference onto itself. Am Math Soc Transl, 1965, 46: 213-284

22 Donato P F A D, Macau E E N, Grebogi C. Phase locking control in the circle map. Nonlin Dyn, 2007, 47: 75-82

23 Pikovsky A, Rosenblum M, Kurths J. Synchronization: A Universal Concept in Nonlinear Sciences. New York: Cambridge University Press, 2001

24 Chen C T. Linear System Theory and Design. New York: Oxford University Press, 1999

25 Sarasola C, Torrealdea F J, D'Anjou A, et al. Cost of synchronizing different chaotic systems. Math Comput Simul, 2002, 58: 309- 327

26 Sarasola C, Torrealdea F J, D'Anjou A, et al. Energy balance in feedback synchronization of chaotic systems. Phys Rev E, 2004, 69: 011606

27 Ma J, Huang L, Xie Z B, et al. Simulated test of electric activity of neurons by using Josephson junction based on synchronization scheme. Commun Nonlin Sci Numer Simul, 2012, 17: 2659-2669

28 Gopfert M C, Robert D. Active auditory mechanics in mosquitoes. Proc R Soc Lond B, 2001, 268: 333-336

29 Warren B, Lukashkin A N, Russell I J. The dynein-tubulin motor powers active oscillations and amplification in the hearing organ of the mosquito. Proc R Soc B, 2010, 277: 1761-1769

Open Access This article is distributed under the terms of the Creative Commons Attribution License which permits any use, distribution, and reproduction in any medium, provided the original author(s) and source are credited. 